\title{
Laser-Beam Zooming to Mitigate Crossed-Beam Energy Losses in Direct-Drive Implosions
}

\author{
I. V. Igumenshchev, ${ }^{1}$ D. H. Froula, ${ }^{1}$ D. H. Edgell, ${ }^{1}$ V. N. Goncharov, ${ }^{1,2}$ T. J. Kessler, ${ }^{1}$ F. J. Marshall, ${ }^{1}$ R. L. McCrory, ${ }^{1,2,3}$ \\ P. W. McKenty, ${ }^{1}$ D. D. Meyerhofer,${ }^{1,2,3}$ D. T. Michel, ${ }^{1}$ T. C. Sangster, ${ }^{1}$ W. Seka, ${ }^{1}$ and S. Skupsky ${ }^{1}$ \\ ${ }^{1}$ Laboratory for Laser Energetics, University of Rochester, 250 East River Road, Rochester, New York 14623-1299, USA \\ ${ }^{2}$ Department of Mechanical Engineering, University of Rochester, Rochester, New York 14623, USA \\ ${ }^{3}$ Department of Physics and Astronomy, University of Rochester, Rochester, New York 14623, USA
}

(Received 19 December 2012; published 1 April 2013)

\begin{abstract}
Spherically symmetric direct-drive-ignition designs driven by laser beams with a focal-spot size nearly equal to the target diameter suffer from energy losses due to crossed-beam energy transfer (CBET). Significant reduction of CBET and improvements in implosion hydrodynamic efficiency can be achieved by reducing the beam diameter. Narrow beams increase low-mode perturbations of the targets because of decreased illumination uniformity that degrades implosion performance. Initiating an implosion with nominal beams (equal in size to the target diameter) and reducing the beam diameter by $\sim 30 \%-40 \%$ after developing a sufficiently thick target corona, which smooths the perturbations, mitigate CBET while maintaining low-mode target uniformity in ignition designs with a fusion gain $\gg 1$.
\end{abstract}

DOI: 10.1103/PhysRevLett.110.145001

PACS numbers: 52.57.Fg, 52.38.Dx, 52.65.Kj, 79.20.Eb

Direct-drive inertial confinement fusion (ICF) uses the energy of multiple laser beams to illuminate and implode a millimeter-scale capsule containing cryogenic nuclear fuel $[1,2]$. Fusion reactions are initiated in the central hot spot when the capsule reaches maximum compression. One of the key physical conditions for ignition (i.e., getting fusion gain $G>1$, where $G$ is the ratio of the fusion energy to laser energy $E_{\mathrm{L}}$ ) is to achieve a high implosion hydrodynamic efficiency $\eta=E_{\mathrm{kin}} / E_{\mathrm{L}}$, which characterizes the conversion of $E_{\mathrm{L}}$ to kinetic energy $E_{\text {kin }}$ of the imploding capsule shell. This condition requires that the shell velocity exceed a minimum threshold value $V_{\text {imp }} \approx 3 \times 10^{7} \mathrm{~cm} / \mathrm{s}$, while maintaining a fuel areal density $\rho R \gtrsim 0.3 \mathrm{~g} / \mathrm{cm}^{2}$ during maximum target compression [3].

Direct-drive implosion experiments are conducted on OMEGA [4] and National Ignition Facility [5] laser systems operating at $\lambda_{\mathrm{L}}=351 \mathrm{~nm}$ with on-target overlapped laser intensities $I_{\mathrm{L}} \sim 10^{14}-10^{15} \mathrm{~W} / \mathrm{cm}^{2}$. The laser absorption in the target corona is dominated by inverse bremsstrahlung. To provide the best illumination uniformity, the focal-spot radius of laser beams $R_{b}$ is taken nearly equal to the target radius $R_{t}, R_{b} / R_{t} \approx 1$ [6]. Here, $R_{b}$ is defined to encircle $95 \%$ of the beam energy. Implosion simulations, assuming energy losses due to only radiation and thermal expansion of the corona, predict $\eta \approx 6 \%$. This hydrodynamic efficiency is sufficient to achieve robust ignition $(G \gg 1)$ in designs using $E_{\mathrm{L}} \gtrsim 1$ MJ $[3,7]$. Recent studies have shown that crossed-beam energy transfer (CBET) [8] resulting from stimulated Brillouin scattering [9] can cause energy losses reducing $\eta$ by $\sim 20 \%-30 \%$ $[10,11]$.

CBET removes energy from incoming light rays that interact with ion-acoustic waves in low-electron-density regions $\left(n_{e} \sim 0.2-0.3 n_{\mathrm{cr}}\right.$, where $n_{\mathrm{cr}}=9 \times 10^{21} \mathrm{~cm}^{-3}$ is the critical density) of the target corona [8]. The stimulated
Brillouin scattering gain responsible for CBET is maximum for incoming center-beam rays and is proportional to the intensity of outgoing rays from edges of opposing beams. Therefore, reducing the intensity at beam edges, or reducing $R_{b}$, reduces CBET [8].

Spherically symmetric implosion experiments on OMEGA employing laser beams with small on-target focal spots (which can be defocused to larger spots) were conducted to study the regime where $R_{b} / R_{t}$ takes the values of 0.5 to 1.1 . The results showed the predicted increases in laser absorption (via scattered-light measurements) and implosion velocity (via bang time and shell trajectory measurements) when reducing $R_{b} / R_{t}$ [10]. An analysis of the uniformity of these implosions performed with the help of self-emission x-ray images found large perturbations with the dominant mode $L=10$ in the case of small $R_{b} / R_{t}$ [10]. The latter finding suggests that small-radius beams introduce significant low-mode perturbations to the targets because of increased illumination nonuniformities (beam overlap). These perturbations reduce the implosion performance and compromise the improvements in laser coupling achieved with small-radius beams.

Spherically symmetric direct-drive-ignition designs suffer from CBET losses, and so, therefore, CBET must be mitigated. For example, simulations predict that a particular 1.5-MJ direct-drive-ignition design [7] fails to ignite when CBET is considered (Fig. 1). Small-radius beams can be employed to mitigate CBET, but these beams introduce significant perturbations to the targets that degrade the implosion performance.

In this Letter, the concept of beam zooming is proposed to help mitigate CBET and maintain good target uniformity. Beam zooming reduces $R_{b} / R_{t}$ from the initial nominal value of 1 to less than 1 after a geometrically thick $\left(\sim R_{t}\right)$ target corona is developed. Before zooming, when the 

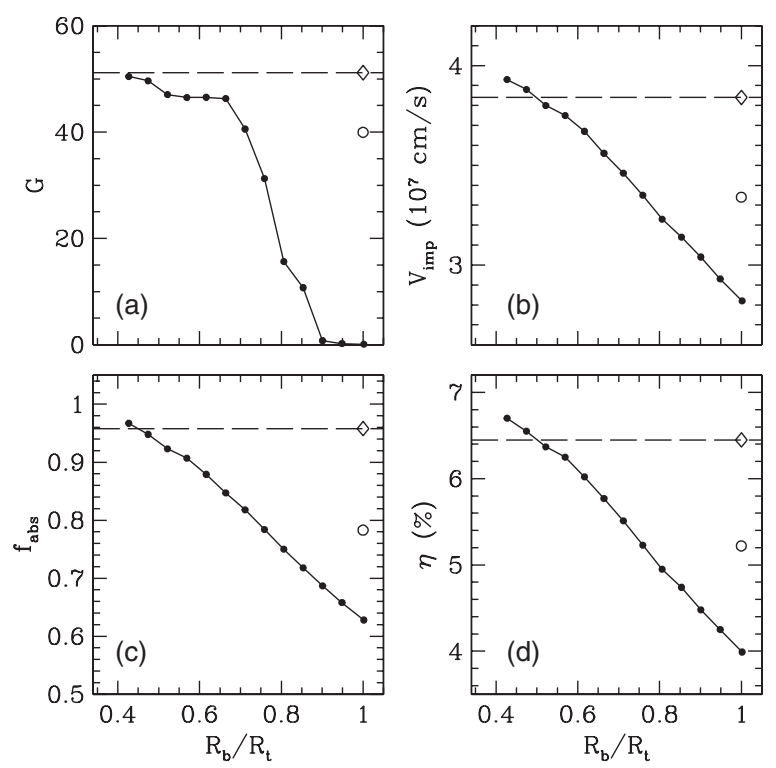

FIG. 1. Simulated (a) fusion gain $G$, (b) implosion velocity $V_{\text {imp }}$, (c) laser-absorption fraction $f_{\text {abs }}$, and (d) implosion hydrodynamic efficiency $\eta$ for the 1.5-MJ spherically symmetric direct-drive implosion design [7] as functions of $R_{b} / R_{t}$. The black circles and solid lines correspond to simulations including CBET. The open diamonds and dashed lines show the performance in the nominal configuration (assuming $R_{b} / R_{t}=1$ ) without CBET. The open circles represent the improvements in the nominal configuration with CBET, when CBET is mitigated employing two-color laser light [8].

corona is geometrically thin and seeding perturbations from beam overlap is most efficient, illuminating targets with nominal-size beams provides the maximum uniformity. After zooming, CBET is mitigated and the thick corona has a sufficiently large conduction zone, which smooths the perturbations.

Figure 1 shows the simulated performance of the $1.5-\mathrm{MJ}$ design in the nominal configuration $\left(R_{b} / R_{t}=1\right)$, which yields a gain $G=52$ when CBET losses are not considered (open diamonds) and does not ignite $(G<1)$ when these losses are considered (black circles). This implosion is simulated using the one-dimensional (1D) ICF hydrodynamic code LILAC [12], which includes a nonlocal thermaltransport model [13]. CBET reduces the laser absorption fraction $f_{\text {abs }}$ from $96 \%$ to $63 \%, V_{\text {imp }}$ from $3.8 \times 10^{7}$ to $2.8 \times 10^{7} \mathrm{~cm} / \mathrm{s}$, and $\eta$ from $6.5 \%$ to $4 \%$. Studies indicate that the loss of energy from CBET in direct-drive-ignition designs cannot be compensated by simply increasing $E_{\mathrm{L}}$. This is because CBET is proportional to the intensity $I_{\mathrm{L}}[8]$ and any increase of $E_{\mathrm{L}}$ (which corresponds to increasing $I_{\mathrm{L}}$ in the fixed-radius targets) causes the proportional increase of CBET losses, making $\eta$ a nearly constant or even a decreasing function of $E_{\mathrm{L}}$. Robust direct-drive-ignition designs benefit from the mitigation of CBET.

Improvements in the $1.5-\mathrm{MJ}$ design with CBET when $R_{b} / R_{t}<1$ are demonstrated in Fig. 1 (black circles). The implosion velocity $V_{\text {imp }}$ [Fig. 1(b)], absorption fraction $f_{\text {abs }}$ [Fig. 1(c)], and hydrodynamic efficiency $\eta$ [Fig. 1(d)] gradually increase with decreasing $R_{b} / R_{t}$. All these quantities approach the values characteristic to the implosion without CBET (open diamonds) at $R_{b} / R_{t} \approx 0.5$. The gain curve shows a cliff around $R_{b} / R_{t}=0.8$ [Fig. 1(a)]. This design is predicted to ignite with gain $G \approx 1$ at $R_{b} / R_{t}=$ 0.9 , and the gain is saturated near the maximum value, $G \approx 50$, at $R_{b} / R_{t}<0.7$. The improvements in the implosion performance that results from reducing $R_{b}$ can be partially attributed to mitigating CBET and partially to more-normal incident illumination of the target [14]. An analysis shows that the latter effect is less important than the improvements from mitigating CBET.

The effect of small-radius beams, which degrade the symmetry of implosion targets, was investigated using the two-dimensional (2D) ICF hydrodynamic code DRACO [15]. DRACO simulations use the cryogenic, lowadiabat ( $\alpha=2$, where $\alpha$ is the ratio of the plasma pressure to Fermi-degenerated pressure), triple-picket OMEGA implosion design shown in Fig. 2. A flux-limited thermal-transport model [16] with a flux limiter $f=0.06$ was employed. These simulations do not include the CBET model, which currently can be applied only in 1D symmetric implosions. A 3D model of CBET coupled with DRACO is being developed [17,18]. Although 2D simulations without CBET and with nominal-size beams are not as accurate, they can provide information about the perturbation levels. The simulations using small-size beams are not significantly affected by the absence of CBET because CBET is mitigated and should be small. The OMEGA laser uses 60 beams that are uniformly distributed over a sphere and arranged in hexagon and pentagon clusters. The laser deposition is calculated using a 3D ray-trace algorithm and the actual OMEGA beam-port geometry. The calculated energy deposition is then azimuthally averaged to be used in axisymmetric DRACO simulations. The current study considers perturbations from the beam overlap but not other laser-induced perturbations
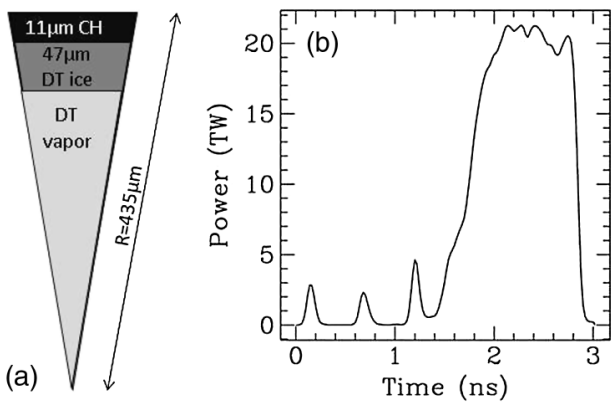

FIG. 2. Low-adiabat $(\alpha=2), 24-\mathrm{kJ}$ triple-picket cryogenic OMEGA implosion design (shot 66613) used in 2D DRACO simulations. (a) Target geometry and (b) laser power history. A spherically symmetric simulation of this design without CBET predicts a yield of $3.4 \times 10^{14}$ neutrons. 
(such as laser imprint, and beam mispointing and mistiming).

Figures 3(a) and 3(b) compare the simulated distributions of density at the moment of maximum neutron production for the two cases of the nominal beams, $R_{b} / R_{t}=1$, and small-radius beams, $R_{b} / R_{t}=0.6$, respectively. In the nominal case, the perturbations are small and dominated by the $L=10$ mode from beam overlap [Fig. 3(a)]. For the small-radius beams, large distortion of the target is apparent [Fig. 3(b)]. The areal density $\Sigma_{\text {rms }}$ at the peak neutron production increases from $7 \%$ to $22 \%$ and the relative neutron yield (2D yield over "clean" $1 \mathrm{D}$ yield [YOC]) decreases from $95 \%$ to $26 \%$ in the nominal and small-radius beam cases, respectively.

Using the nominal-size beams at the beginning of the implosion and zooming beam focal spots to smaller size after developing a geometrically thick $\left(\sim R_{t}\right)$ corona around the target helps to reduce perturbations from beam-overlap nonuniformities and, simultaneously, mitigates CBET. There are two reasons this technique is effective: First, the perturbations from beam overlap are developed mainly at the beginning of the laser pulse. The corona developed during the main laser drive forms an extended conduction zone. This zone is capable of efficiently smoothing perturbations from the laser, including the relatively large scale perturbations induced by beam overlap. The geometrically thin corona existing at the beginning of the pulse cannot provide such a smoothing. Second, the study of CBET in OMEGA implosions showed that CBET is relatively weak at the beginning of the drive and takes its maximum value later in time, when the

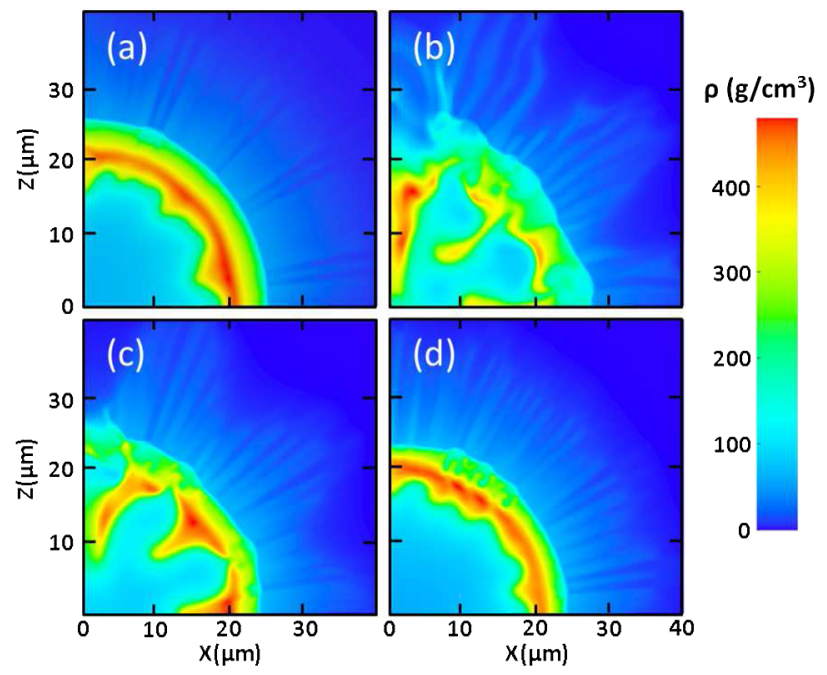

FIG. 3 (color online). Simulated distributions of the density at the peak neutron production for the implosion design shown in Fig. 2. (a) Nominal-size beams, $R_{b} / R_{t}=1$; (b) reduced-size beams, $R_{b} / R_{t}=0.6$; (c) zooming beams from $R_{b} / R_{t}=1$ to 0.6 at $t=0.4 \mathrm{~ns}$ [after the first picket; see Fig. 2(b)]; (d) same zooming beams as in (c), but at $t=0.9 \mathrm{~ns}$ (after the second picket). extended corona is developed [8]. Therefore, using the nominal laser beams $\left(R_{b} / R_{t}=1\right)$ at the beginning of implosions is not critical with respect to mitigating CBET and provides the best illumination uniformity at this critical moment when perturbations from the laser are most efficiently imprinted into the target. The latertime reduction of the beam diameter helps to mitigate CBET during the main drive, whereas the corresponding increase of beam-overlap illumination nonuniformities does not introduce significant perturbations to the target due to the smoothing effect of the corona.

The moment of zooming should be chosen to balance the reduction of target nonuniformities and mitigation of CBET. Zooming too early can compromise the uniformity and zooming too late can result in excessive energy losses related to CBET. Figures 3(c) and 3(d) show DRACO simulation results assuming a two-state zooming from $R_{b} / R_{t}=$ 1 to 0.6 at two different moments, $t=0.4 \mathrm{~ns}$ (after the first picket) and $0.9 \mathrm{~ns}$ (after the second picket), respectively. The target is significantly distorted when zooming occurs just after the first picket, indicating that the target corona is not developed enough to smooth the perturbations. Zooming the beams after the second picket shows significant improvements in target uniformity. Zooming even later, after the third picket, shows about the same improvements as zooming after the second picket. Table I summarizes the simulation results showing YOC and areal density $\Sigma_{\text {rms }}$ for the models assuming different zooming options: zooming after the first, second, and third pickets, and no zooming. These data indicate that the decrease of $\Sigma_{\text {rms }}$ in the models with delayed zooming is anticorrelated with the increase of YOC. Zooming after the second and third pickets shows about the same relative implosion performance as in the case where the nominal-radius beams are used without zooming.

Potential schemes to implement zooming of the focal spot on target involve modifications to the spatial coherence of the laser that cause broadening in the beam's far field [19]. The most practical method for implementing zooming on modern laser systems (e.g., OMEGA and National Ignition Facility) appears to be time-dependent phase conversion. A new phase-plate design, referred to as a zooming phase plate (ZPP), is proposed. ZPPs will have a radial phase transition where the central area will produce a larger, low-order Gaussian focal spot, while the outer area will produce a smaller, high-order Gaussian focal spot.

TABLE I. Summary of DRACO simulation results.

\begin{tabular}{lccc}
\hline \hline Zooming & $R_{b} / R_{t}$ & YOC $(\%)$ & $\Sigma_{\text {rms }}(\%)$ \\
\hline Not applied & 0.6 & 26 & 22 \\
After first picket & 1.0 to 0.6 & 55 & 20 \\
After second picket & 1.0 to 0.6 & 89 & 6 \\
After third picket & 1.0 to 0.6 & 88 & 9 \\
Not applied & 1.0 & 95 & 7 \\
\hline \hline
\end{tabular}


This configuration requires a smaller-diameter beam during the pickets and a midsection cutout of the near field during the drive pulse [20].

The beam-zooming technique can benefit from implementing it into a two-color laser system. This system alone can reduce CBET by a factor of $\sim 2$ (see open circles in Fig. 1) if the wavelength separation of the colors $\Delta \lambda>5 \AA$ (for $\lambda_{\mathrm{L}}=351 \mathrm{~nm}$ ) [8]. Simultaneously employing the beam zooming and two-color techniques will make it possible to use less-restrictive requirements for the zooming ratio and, therefore, improve implosion uniformity while maintaining low CBET.

The beam-zooming technique applies to spherically symmetric illumination and might not be applicable to the polar-drive configuration [21]. Mitigation strategies optimized for polar drive are under investigation $[17,18]$.

In summary, CBET reduces the implosion velocity in directly driven targets below the ignition-relevant values and must be mitigated to obtain robust direct-drive-ignition designs. Implosion experiments on OMEGA demonstrated that laser beams with on-target focal spots smaller than the target diameter mitigate CBET. Simulations suggest that using beams with $R_{b} / R_{t}<0.7$ in the 1.5 -MJ direct-driveignition design recover the majority of the implosion velocity lost to CBET. Such a reduction of $R_{b} / R_{t}$ introduces large-amplitude, low-mode perturbations to the targets because of increasing beam-overlap illumination nonuniformities. Experiments and simulations indicate that these perturbations degrade the implosion performance. Beam zooming is proposed to overcome this problem. Employing beams with the nominal $R_{b} / R_{t}=1$ at the beginning of the laser pulse and later reducing the beam radius to $R_{b} / R_{t}<$ 0.7 during the main drive can improve low-mode uniformity of the target while maintaining low-energy losses relating to mitigating CBET. The performance of $2 \mathrm{D}$ axisymmetric OMEGA implosions assuming perturbations from optimally zooming beams is predicted to be almost as good as the performance of the corresponding 1D, spherically symmetric implosions. The practical way of implementing beam zooming is considered using ZPPs.

The analysis of perturbations performed in this Letter is limited only to 2D axisymmetric modes and does not include the effects of CBET. It is unlikely that more accurate 3D simulations including CBET will change the qualitative conclusions of this analysis, but some quantitative changes are expected. For example, 3D perturbations will grow faster and introduce more damage to implosions. Further developments of the zooming technique will require investigating other laser-induced perturbations attributable to ZPPs (e.g., laser imprint). These issues will be addressed in future studies.

This work was supported by the U.S. DOE Office of Inertial Confinement Fusion under Cooperative Agreement No. DE-FC52-08NA28302, the University of Rochester, and the New York State Energy Research and Development Authority. The support of DOE does not constitute an endorsement by DOE of the views expressed in this article.

[1] J. D. Lindl, Inertial Confinement Fusion (Springer, New York, 1998), pp. 39, 61.

[2] R. L. McCrory et al., Phys. Plasmas 15, 055503 (2008).

[3] S. Atzeni and J. Meyer-ter-Vehn, The Physics of Inertial Fusion: Beam Plasma Interaction, Hydrodynamics, Hot Dense Matter, International Series of Monographs on Physics (Clarendon, Oxford, 2004), pp. 31-46.

[4] T. R. Boehly, D. L. Brown, R. S. Craxton, R. L. Keck, J. P. Knauer, J.H. Kelly, T. J. Kessler, S. A. Kumpan, S. J. Loucks, S. A. Letzring, F. J. Marshall, R. L. McCrory, S. F. B. Morse, W. Seka, J. M. Soures, and C.P. Verdon, Opt. Commun. 133, 495 (1997).

[5] J.A. Paisner, J.D. Boyes, S. A. Kumpan, W. H. Lowdermilk, and M.S. Sorem, Laser Focus World 30, 75 (1994).

[6] S. Skupsky and K. Lee, J. Appl. Phys., 54, 3662 (1983).

[7] V. N. Goncharov, T. C. Sangster, T. R. Boehly, S. X. Hu, I. V. Igumenshchev, F. J. Marshall, R. L. McCrory, D. D. Meyerhofer, P. B. Radha, W. Seka, S. Skupsky, C. Stoeckl, D. T Casey, J. A. Frenje, and R. D. Petrasso, Phys. Rev. Lett. 104, 165001 (2010).

[8] I. V. Igumenshchev, D. H. Edgell, V. N. Goncharov, J. A. Delettrez, A. V. Maximov, J. F. Myatt, W. Seka, A. Shvydky, S. Skupsky, and C. Stoeckl, Phys. Plasmas 17, 122708 (2010).

[9] W.L. Kruer, The Physics of Laser Plasma Interactions (Addison-Wesley, Reading, MA, 1988), pp. 46, 88.

[10] D. H. Froula, I. V. Igumenshchev, D. T. Michel, D. H. Edgell, R. Follett, V. Yu. Glebov, V. N. Goncharov, J. Kwiatkowski, F. J. Marshall, P. B. Radha, W. Seka, C. Sorce, S. Stagnitto, C. Stoeckl, and T. C. Sangster, Phys. Rev. Lett. 108, 125003 (2012).

[11] I. V. Igumenshchev et al., Phys. Plasmas 19, 056314 (2012).

[12] J. Delettrez, R. Epstein, M.C. Richardson, P. A. Jaanimagi, and B.L. Henke, Phys. Rev. A 36, 3926 (1987).

[13] V. N. Goncharov et al., Phys. Plasmas 15, 056310 (2008).

[14] J.H. Gardner and S.E. Bodner, Phys. Fluids 29, 2672 (1986).

[15] D. Keller, T. J. B. Collins, J. A. Delettrez, P. W. McKenty, P. B. Radha, B. Whiney, and G. A. Moses, Bull. Am. Phys. Soc. 44, 37 (1999).

[16] R. C. Malone, R. L. McCrory, and R. L. Morse, Phys. Rev. Lett. 34, 721 (1975).

[17] D.H. Edgell, R.E. Bahr, V.N. Goncharov, I. V. Igumenshchev, J. F. Myatt, P. B. Radha, W. Seka, and D. H. Froula, Cross-Beam Energy Transfer in PolarDrive Implosions on OMEGA, LLE Review Quarterly Report 133 (Laboratory for Laser Energetics, University of Rochester, Rochester, NY, 2013).

[18] J. A. Marozas and T. J. B. Collins, Bull. Am. Phys. Soc. 54, 344 (2012). 
[19] M. C. Myers, J. D. Sethian, J. L. Giuliani, R. Lehmberg, P. Kepple, M. F. Wolford, F. Hegeler, M. Friedman, T.C. Jones, S. B. Swanekamp, D. Weidenheimer, and D. Rose, Nucl. Fusion 44, S247 (2004).

[20] D. H. Froula, T. J. Kessler, I. V. Igumenshchev, A. Shvydky, J.H. Kelly, J.D. Zuegel, E. Hill, and V. N. Goncharov, "Mitigation of Cross-Beam Energy Transfer:
Implications of Two-State Optical Zooming on OMEGA" (to be published).

[21] S. Skupsky, J. A. Marozas, R. S. Craxton, R. Betti, T. J. B. Collins, J. A. Delettrez, V. N. Goncharov, P. W. McKenty, P. B. Radha, T. R. Boehly, J. P. Knauer, F. J. Marshall, D. R. Harding, J. D. Kilkenny, D. D. Meyerhofer, T. C. Sangster, and R. L. McCrory, Phys. Plasmas 11, 2763 (2004). 